\title{
Binocular rivalry alternations and their relation to visual adaptation
}

\section{Daphne Roumani* and Konstantinos Moutoussis}

Cognitive Science Division, Department of Philosophy and History of Science, University of Athens, Athens, Greece

\section{Edited by:}

Alexander Maier, Vanderbilt

University, USA

Reviewed by:

Raymond Van Ee, University

Utrecht, Netherlands

Chris Paffen, Utrecht University,

Netherlands

\section{*Correspondence:}

Daphne Roumani, Cognitive Science

Laboratory, Department of

Philosophy and History of Science,

University of Athens, University

Campus, Ano llissia, Athens 15771

Greece.

e-mail:droumani@phs.uoa.gr
When different stimuli are presented dichoptically, perception alternates between the two in a stochastic manner. After a long-lasting and rigorous debate, there is growing consensus that this phenomenon, known as binocular rivalry (BR), is the result of a dynamic competition occurring at multiple levels of the visual hierarchy. The role of low- and high-level adaptation mechanisms in controlling these perceptual alternations has been a key issue in the rivalry literature. Both types of adaptation are dispersed throughout the visual system and have an equally influential, or even causal, role in determining perception. Such an explanation of BR is also in accordance with the relationship between the latter and attention. However, an overall explanation of this intriguing perceptual phenomenon needs to also include noise as an equally fundamental process involved in the stochastic resonance of perceptual bistability.

Keywords: binocular rivalry, adaptation, perceptual alternations

\section{INTRODUCTION}

Binocular Rivalry (BR) is the perceptual competition resulting when two different images are presented simultaneously to corresponding retinal locations (Wheatstone, 1838). A "race" between the two monocular images leads to continuous perceptual alternations, with successive periods of dominance and suppression. The spontaneous character of these alternations (Levelt, 1965) is the hallmark of rivalry, and a rigorous debate regarding its origin is whether it is based on competition between the two eyes or between the two stimuli (Blake and Logothetis, 2002). The traditional version of this debate has been challenged by compelling evidence supporting that rivalry involves a cascade of neuronal events spanning through multiple levels of the visual hierarchy, thus, favoring an integrative approach (see Sterzer et al., 2009; Blake and Wilson, 2011). However, the interplay between lowlevel and high-level visual processing as well as their exact role in the instigation of rivalry remains to be clarified. Several attempts to shed light on this question focus on adaptation as a crucial variable (Alais et al., 2010; Kang and Blake, 2010; Theodoni et al., 2011a).

Adaptation is a ubiquitous property of the visual system (Clifford et al., 2007) and has been used as a powerful tool for dissecting the involvement of high-level, feature-based, and lower-level, eye-based factors in BR (e.g., Blake et al., 2006; van Boxtel et al., 2008; Lin and He, 2009; Bartels and Logothetis, 2010). It has in this way contributed in establishing a multi-level localization of rivalry (Wilson, 2003). Furthermore, adaptation per se could be the driving force behind spontaneous perceptual alternations (e.g., Kang and Blake, 2010). The present review aims to bring together evidence supporting the idea that adaptation is a key constituent process in generating perceptual switches which, together with other important constraints such as noise and voluntary control (Kim et al., 2006; van Ee, 2009; Paffen and Alais,
2011), could lead to a complete and successful explanation of this intriguing perceptual phenomenon.

\section{THE POTENTIAL ROLE OF ADAPTATION IN TRIGGERING PERCEPTUAL ALTERNATIONS LOCAL VERSUS GLOBAL ADAPTATION}

An adaptation-based explanation of BR was firstly introduced in early, interocular-competition theories, which assigned a causal role to early, local inhibitory mechanisms (Blake, 1989). Stimulus-competition theories, on the other hand, have treated perceptual alternations as the result of a perceptual inference process, which controls these early mechanisms via feedback from higher visual areas (Logothetis, 1998; Leopold and Logothetis, 1999). Both views thus accept a crucial role for adaptation, making pertinent the need to clarify the stage at which adaptationbased interactions trigger bistability. Could adaptation provide a causal explanation for BR? If so, is it local or global adaptation processes that enjoy a primary role in triggering these perceptual switches? With the term local adaptation, we refer to adaptation occurring at an early, perhaps monocular level that is governed by local, eye-based processes. Global adaptation, on the other hand, refers mostly to feature/pattern-based processing at later processing stages of the visual system, with neurons having more complex receptive field properties (Alais and Blake, 1998). Such a distinction is consistent with the fact that suppression-depth is stronger for complex stimuli (Alais and Melcher, 2007), the former also probably increasing as one ascends the stages of visual hierarchy (Nguyen et al., 2003). Besides, it is well-established that low- and high-level rivalry are differentially affected by attention (Meng and Tong, 2004; van Ee et al., 2005) or noise (van Ee, 2009) and, despite the fact that they might share a common computational mechanism (Klink et al., 2008b), seem to take place at different processing stages (Wilson, 2003). 
Given the multi-stage localization of both rivalry and adaptation (Clifford et al., 2007), the crucial question concerns how adaptation mechanisms at different levels interact in order to produce a perceptual change. It has been suggested that perceptual switches arise from adaptation of the currently dominant neuronal representation, the latter being gradually weakened via competing inhibition from the antagonistic representation, eventually succumbing to suppression that leads to a perceptual switch (Kang and Blake, 2010). These interactions are thought to occur at an early processing level (Blake, 1989; Wilson, 2003) even though mutual inhibition may rise from multiple cortical areas as well (Seely and Chow, 2011). Accordingly, initial dominance biases at specific locations of the visual field can be broken down by preceding spatiotopic adaptation, indicating that periods of adaptation can induce local perceptual switches (Carter and Cavanagh, 2007). Furthermore, the longer the duration of adaptation, the later the first breakout from suppression (van Ee, 2011), supporting a model of competing neuronal populations which encode the two alternative stimulus interpretations (Wilson, 2003). The spatial "profile" of this escape from suppression takes place in an inhomogeneous manner, arising from local random differences in adaptation at the monocular processing level, which might determine local perceptual switches (van Ee, 2011). A similar conclusion about the primacy of local adaptation comes from a study by Alais and Melcher (2007), focusing on the coherence of rivalry alternations (vs. piecemeal rivalry) and the suppressiondepth of complex (faces) and simple (gratings) stimuli. They have shown that global adaptation processes are unable to overcome the stochastic character of rivalry alternations in local zones, even when a complex stimulus is rivaling with a simple one. These findings suggest that global processes fail to exert a stabilizing influence in rivalry alternations, thus assigning a primary role to local adaptation processes (but see van Boxtel et al., 2008). Along the same lines, Carlson and He (2004) have also shown that, unless there are local incompatibilities between rivaling stimuli, global differences alone fail to produce rivalry. Results like these do reject a role of global, top-down influences in rivalry, but rather suggest that it is local conflict that counts more. Contrary to these findings, other studies suggest that perceptual differences can induce rivalry despite physical similarities: physically identical random-dot displays, which are phenomenally different due to adaptation, can engage in rivalry (Blake et al., 1998), and BR can be induced by identical stimuli within a different chromatic surround (Andrews and Lotto, 2004). Thus, the emerging question concerns the conditions which are sufficient for bistability to be triggered: could perceptual differences account for the perceptual alternations or is the eye-of-origin information the driving force?

By interrupting rivalry at various time points after a period of dominance, Bartels and Logothetis (2010) have found a strong eye-of-origin stabilizing effect, which declines over time and a higher-level image/perceptual contribution which initially exhibits a small stabilizing effect, but later strongly destabilizes perception. This complementary variation over time underlines the need to include both eye-based and stimulus-based processes in a rivalry account, with the latter influencing eye-based processes possibly via feedback signals. Along these lines, recent findings show a weak modulation of early visual cortex activity by perception (Keliris et al., 2010), which becomes stronger in higher areas, as reported previously (Leopold and Logothetis, 1996). In a similar vein, Stuit et al. (2011) have examined the differential contribution of eye- and stimulus-factors as potential grouping cues in synchronizing the dominance of spatially segregated visual targets (Kovacs et al., 1996). They have shown that, even though both kind of cues act independently to promote grouping, the eye-of-origin information has a superior value as a grouping-cue. Taken together, these findings suggest that both levels of processing should be included in an adaptation-based explanation of BR. Nevertheless, it is rather difficult to separate the contribution of eye-based and feature-based processes in BR even at the earliest stage of the competition since, in addition to eye-of-origin signals, single cells in V1 also contain feature-related information (see Keliris et al., 2010) and top-down processing can influence the very early stage of rivalry onset (Klink et al., 2008a).

\section{LOCATING CAUSALITY WITHIN THE CORTEX}

It is obvious that unraveling the contribution of eye- and stimulus-related factors is still of high importance in rivalry literature (see Paffen and Alais, 2011), with serious implications regarding the neural localization of the triggering point of alternations. Accepting the primacy of image-content information, results in treating alternations as the outcome of inferential processes that concern perceptual interpretations (Sterzer et al., 2009). In this context, perceptual changes could be an expression of the frequent re-evaluation of the interpretations of the sensory input (Leopold and Logothetis, 1999). Rivalry starts because perceptual conflict about meaning is maximized (Sterzer et al., 2009), rather than just because fusion fails (Wolfe, 1986; Blake, 1989). The idea is further supported by imaging data, showing prefrontal and parietal activation during perceptual rivalry transitions (Lumer et al., 1998; Zaretskaya et al., 2010). Activation of right inferior frontal cortex is shown to have a temporal precedence, thus suggesting a causal role to these areas in the initiation of perceptual changes (Sterzer and Kleinschmidt, 2007). Whether these findings also indicate that top-down processes initiate the reorganization of activity in early visual cortex (Sterzer et al., 2009), remains speculative. If so, the adaptation of early, local inhibitory processes could play a role in destabilizing the activity underpinning the currently dominant percept, thus driving higher-order evaluative processes to initiate a perceptual reorganization (Sterzer and Kleinschmidt, 2007). Although such a scenario would give primacy to global adaptation mechanisms, this "trigger hypothesis" has been recently challenged by evidence toward the opposite direction (Knapen et al., 2011). By using more realistic rivalry simulations in the rivalry-mimicking condition, ${ }^{1}$ no difference was found in frontoparietal activation between endogenous (actual rivalry) and exogenous (replay) transitions, with a difference observed in occipital cortex alone (see figure 3 in Knapen et al., 2011). This pattern of results implies that changes observed in frontoparietal activity may be the consequence of alternations rather than their cause, possibly reflecting

\footnotetext{
${ }^{1}$ As opposed to earlier studies, using instantaneous replays that badly mimic the gradual perceptual changes observed in rivalry (e.g., Lumer et al., 1998; Polonsky et al., 2000).
} 
changes in sensory experience, attentional state or task demand processes (Knapen et al., 2011). The existence of a broad network involved causally in BR transitions has been challenged by Kamphuisen et al. (2008). Using an MEG frequency tagging method in order to track how stimulus-based activity relates to perception, they have located the source of rivalry alternations mainly in early visual areas of the occipital lobe. In accordance, Pearson et al. (2007) have provided evidence for a causal role of early visual cortex activity in BR: they have found that TMS applied over V1/V2 during conventional and swap/stimulus rivalry can induce perceptual changes in the former but not in the latter case. On the other hand, TMS applied in parietal cortex during BR results in disrupting alternations rhythm by lengthening dominance durations, implying that causality could be located in higher areas as well (Zaretskaya et al., 2010; see also Paffen and Alais, 2011 for a review on the controversial role frontoparietal areas in $\mathrm{BR})$.

\section{THE ROLE OF ATTENTION}

Although a possible causal role of frontoparietal cortex in BR alternations has not been yet firmly established, the findings mentioned above suggest a tight link between BR and attention, since there seems to be a major topographical overlap between the cortical networks mediating both phenomena (Paffen and Alais, 2011). The susceptibility of the alternation rate to attentional manipulations has been widely used as an indicator of top-down influences in BR, even though its sensitivity as a measure has been doubted (Meng and Tong, 2004). Alternations are enhanced once attention is driven to the stimuli and attenuated when it is withdrawn (Meng and Tong, 2004; van Ee et al., 2005; Paffen et al., 2006), or even eliminated by the total absence of attention (Zhang et al., 2011-but see Roeber et al., 2011). Attentional changes have been long suggested as the cause of alternations, a coreissue on the rivalry debate since its rediscovery by Wheatstone (1838; see also Blake and Logothetis, 2002). Nevertheless, though well-established, the role of voluntary control in BR is clearly limited (Paffen and Alais, 2011), especially when it comes to rivalry between low-level stimuli which, compared to ambiguous figures and rivalry between more complex stimuli, seems to be less subjective to (endogenous) attention (Meng and Tong, 2004; van Ee et al., 2005) and more prone to noisy variations (see van Ee, 2009).

The dependency of BR on bottom-up factors such as stimulus strength on the one hand and attention on the other, might seem contradictory, but could perhaps be explained by evidence for the apparent but quantifiable effects of attention on low-level stimulus characteristics (van Ee et al., 2005; Paffen et al., 2006; Klink et al., 2008b). Attention seems to boost the effective contrast of the attended stimulus (Carrasco et al., 2004; Chong and Blake, 2006), which in turn affects both the alternation rate and the dominance durations, following Levelt's predictions (see Paffen et al., 2006; Klink et al., 2008b). Within this framework, attention may be the counterpart of adaptation, attenuating the buildup of the latter by preventing its effects from developing fully, similar to the way in which stimulus strength's manipulations directly affect the time course of the recovery from adaptation (Wilson, 2003). In order to directly address the influence of adaptation on perceptual switches, Kang and Blake (2010) have created a novel "on-line" adaptation paradigm by physically removing and later reintroducing the suppressed stimulus, so that brief periods of monocular adaptation are interspersed during the actual duration of BR. They have shown that dominance duration decreases with increasing adaptation duration (see also van Ee, 2011). Furthermore, Blake et al. (2003) have found that when the conflicting stimuli are made to move continuously across the retina, alternations slow down significantly-a result also attributed to the removal of local adaptation. The similarity between attentional and adaptational manipulations, suggests that attention is another important variable that has to be incorporated in models explaining rivalry dynamics. Such an account must also take into consideration new experimental evidence showing that such an effect can also be multimodal (e.g., van Ee et al., 2009; Zhou et al., 2010; Chen et al., 2011). It is perhaps worth mentioning here that perceptual stabilization caused by intermittent presentation of rivalrous stimuli (Leopold et al., 2002), an extreme version of lengthening dominance durations (van Ee et al., 2005), has also been explained as the result of the insufficient development of the inhibitory-adaptational interactions eventually leading to a perceptual transition (Chen and $\mathrm{He}, 2004)^{2}$. However, it has been come evident by psychophysical and computational studies that local adaptation (or its absence) cannot account for stabilization caused by intermittent presentation (see Pearson and Clifford, 2005; Brascamp et al., 2007, 2009; Noest et al., 2007; also Pearson and Brascamp, 2008 for a detailed review).

\section{CAN ADAPTATION FULLY EXPLAIN PERCEPTUAL SWITCHES? THE ROLE OF NOISE}

The studies reviewed above make an adaptation-based explanation of rivalry highly plausible. Still, there are predictions of the adaptation-cross inhibition model that a solely adaptation-based account cannot explain (Alais et al., 2010; Kang and Blake, 2010). For example, according to this model, visual sensitivity should change over time in a way that reflects the reciprocal changes in the adaptation level of the antagonistic neuronal populations (Noest et al., 2007). Although earlier studies have failed to support this idea (e.g., Fox and Check, 1972), Alais et al. (2010) have used a novel probe-method to show that sensitivity during both rivalry states is not stable: performance during dominance declines over time, while the opposite is true during suppression. This complementary variation in dominance and suppression sensitivity over time corresponds exactly with the gradual reciprocal changes predicted by the adaptation-inhibition model. This finding, for the first time experimentally confirmed by Alais et al., overcomes what was previously thought an inconsistency between theory and observed data, supporting further the idea of adaptation as a crucial mechanism in BR (Alais et al., 2010).

Another intriguing aspect of $\mathrm{BR}$ is the irregular and unpredictable character of dominance shifts observed in other multistable perceptual phenomena as well (Leopold and Logothetis, 1999; Gigante et al., 2009). This random nature of perceptual

\footnotetext{
${ }^{2}$ Instead of assuming a perceptual memory as it was originally suggested (Leopold et al., 2002).
} 
fluctuations, thought to stem from stochastic variation (Lehky, 1995; Kim et al., 2006), contradicts the fairly regular character of alternations that should be observed if transitions were mediated solely by deterministic processes such as adaptation and cross inhibition (Shpiro et al., 2009; van Ee, 2009; Kang and Blake, 2010). Therefore, noise has also been incorporated in computational models of adaptation-inhibition based perceptual decisions, in order to account for the irregular character of perceptual alternations (e.g., Wilson, 2003, 2007; Noest et al., 2007; Shpiro et al., 2009). It has been suggested that it exerts a crucial, perhaps dominating role (Brascamp et al., 2006; Lankheet, 2006). Stochastic resonance is a noisy-driven process with the virtue of allowing for deterministic (adaptational) influences to take place as well, as in the case of rivalry (van Ee, 2009). It seems to be the key-process emerging from the coupling between adaptation, inhibition, and noise, controlling perceptual transitions (Kim et al., 2006). It has been suggested that it is noise in the adaptation of percept-related neurons, not noise in the crossinhibition neurons, which accounts for transitions (Kim et al., 2006; van Ee, 2009; Theodoni et al., 2011a). This seems to be also true for the serial correlations observed between successive dominance durations, once experimental impurities are controlled for (van Ee, 2009; see also Kang and Blake, 2010). In support of this notion, recent computational studies of the relative contribution of noisy-driven or adaptation-driven models in generating bistability, have shown that noise is a crucial variable that needs to operate in balance with adaptation in generating perceptual alternations (Shpiro et al., 2009; Theodoni et al., 2011a). Other computational models examining the occurrence of alternations during intermittent viewing, go further and incorporate higherorder variables such as perceptual memory of the type Leopold et al. (2002) have suggested (Wilson, 2007; Brascamp et al.,

\section{REFERENCES}

Alais, D., and Blake, R. (1998). Interactions between global motion and local binocular rivalry. Vision Res. 38, 637-644.

Alais, D., Cass, J., O'Shea, R. P., and Blake, R. (2010). Visual sensitivity underlying changes in visual consciousness. Curr. Biol. 20, 1362-1367.

Alais, D., and Melcher, D. (2007). Strength and coherence of binocular rivalry depends on shared stimulus complexity. Vision Res. 47, 269-279.

Andrews, T. J., and Lotto, R. B. (2004). Fusion and rivalry are dependent on the perceptual meaning of visual stimuli. Curr. Biol. 14, 418-423.

Bartels, A., and Logothetis, N. K. (2010). Binocular rivalry: a timedependence of eye and stimulus contributions. J. Vis. 10, 1-14.

Blake, R. (1989). A neural theory of binocular rivalry. Psychol. Rev. 96, 145-167.

Blake, R., and Logothetis, N. (2002). Visual competition. Nat. Rev. Neurosci. 13, 1-11.

2009), as well as voluntary control (Klink et al., 2008a). A recent computational model simulating perceptual decisions regarding higher-order, ambiguous visual stimuli (face/hand) has suggested that adaptation-driven transitions due to afterhyperpolarization currents, can explain oscillatory perceptual alternations better than noise-driven transitions, which are due to the probabilistic spike times of neurons (Theodoni et al., 2011b). It becomes clear that none of these factors should be excluded from a complete explanation of rivalry alternations. When it comes to causality, however, adaptation and noise seem to offer the most plausible explanation.

\section{CONCLUDING REMARKS}

After almost two centuries of rigorous debate about the contribution of eye- and stimulus-factors in rivalry dynamics, there is now growing consensus in incorporating both levels of processing in a successful explanation of the phenomenon (Blake and Wilson, 2011). Adaptation, conceived as a property of both early and late stages of visual processing (Clifford et al., 2007), clearly has an important role in this interaction as a key-point process behind perceptual alternations. Local adaptation is assigned a primary or even causal role in determining rivalry transitions (e.g., Blake, 1989; Pearson et al., 2007; Kang and Blake, 2010). However, this does not rule out the possibility that global adaptation plays an executive role as well, exerting a feedback influence on local inhibitory interactions, thus initiating a perceptual change (e.g., Sterzer et al., 2009; Zhang et al., 2011). Therefore, a model including dynamic interactions between local and global adaptation mechanisms, as well as the dynamics of noise in the observed stochastic variability (Brascamp et al., 2006; van Ee, 2009; Theodoni et al., 2011a), seems to be the ideal substrate for explaining perceptual transitions during BR.

Brascamp, J. W., van Ee, R., Noest, A. J., Jacobs, R. H. A. H., and van den Berg, A. V. (2006). The time course of binocular rivalry reveals a fundamental role of noise. J. Vis. 6 , 1244-1256.

Carlson, T. A., and He, S. (2004). Competing global representations fail to initiate binocular rivalry. Neuron 43, 907-914.

Carrasco, M., Ling, S., and Read, S. (2004). Attention alters appearance. Nat. Neurosci. 7, 308-313.

Carter, O., and Cavanagh, P. (2007). Onset rivalry: brief presentation isolates an early independent phase of perceptual competition. PLOS One 2:e343. doi: 10.1371/journal.pone.0000343

Chen, Y. C., Yeh, S. L., and Spence, C. (2011). Crossmodal constraints on human perceptual awareness: auditory semantic modulation of binocular rivalry. Front. Psychol. 2:212. doi: 10.3389/fpsyg.2011. 00212

Chen, X., and He, S. (2004). Local factors determine the stabilization of monocular ambiguous and binocular rivalry stimuli. Curr. Biol. 14, 1013-1017.

Chong, S. C., and Blake, R. (2006). Exogenous attention and endogenous attention influence initial dominance in binocular rivalry. Vision Res. 46, 1794-1803.

Clifford, C. W., Webster, M. A., Stanley, G. B., Stocker, A. A., Kohn, A., Sharpee, T. O., and Schwartz, O. (2007). Visual adaptation: neural, psychological and computational aspects. Vision Res. 47, 3125-3131.

Fox, R., and Check, R. (1972). Independence between binocular rivalry suppression duration and magnitude of suppression. J. Exp. Psychol. 93, 283-289.

Gigante, G., Mattia, M., Braun, J. and Del Giudice, P. (2009). Bistable perception modeled as competing stochastic integrations at two levels. PLoS Comput. Biol. 5:e1000430. doi: 10.1371/journal.pcbi.1000430

Kamphuisen, A. P., Bauer, M., and van Ee, R. (2008). No evidence for widespread synchronized networks in binocular rivalry: MEG 
frequency tagging entrains primarily early visual cortex. J. Vis. 8, 4.1-4.8.

Kang, M. S., and Blake, R. (2010). What causes alternations in dominance during binocular rivalry. Atten. Percept. Psychophys. 72, 179-186.

Keliris, G., Logothetis, N., and Tolias, A. (2010). The role of primary visual cortex in perceptual suppression of salient visual stimuli. J. Neurosci. 30, 12353-12365.

Kim, Y-J., Grabowecky, M., and Suzuki, S. (2006). Stochastic resonance in binocular rivalry. Vision Res. 46, 392-406.

Klink, P. C., van Ee, R., Nijs, M. M., Brouwer, G. J., Noest, A. J., and van Wezel, R. J. A. (2008a). Early interactions between neuronal adaptation and voluntary control determine perceptual choices in bistable vision. J. Vis. 8, 16.1-18.

Klink, P. C., van Ee, R., and van Wezel, R. J. A. (2008b). General validity of Levelt's propositions reveals common computational mechanisms for visual rivalry. PLoS One 3:e3473. doi: 10.1371/journal.pone.0003473

Knapen, T., Brascamp, J., van Ee, R., and Blake, R. (2011). The role of frontal and parietal areas in bistable perception. J. Neurosci. 31, 10293-10301.

Kovacs, I., Papathomas, T. V., Yang, M., and Fehır, A. (1996). When the brain changes its mind: interocular grouping during binocular rivalry. Proc. Natl. Acad. Sci. U.S.A. 93, 15508-15511.

Lankheet, M. (2006). Unraveling adaptation and mutual inhibition in rivalry. J. Vis. 6, 304-310.

Lehky, S. R. (1995). Binocular rivalry is not chaotic. Proc. Biol. Sci. 259, 71-176.

Leopold, D., and Logothetis, N. (1996). Activity changes in early visual cortex reflect monkeys' percepts during binocular rivalry. Nature 379, 549-553.

Leopold, D., and Logothetis, N. (1999). Multistable Phenomena: changing views in perception. Trends Cogn. Sci. 3, 254-264.

Leopold, D. A., Wilke, M., Maier, A., and Logothetis, N. K. (2002). Stable perception of visually ambiguous patterns. Nat. Neurosci. 5, 605-609.

Levelt, W. (1965). On Binocular Rivalry. Soesterberg, Netherlands: Institute for Perception RVO-TNO.
Lin, Z., and He, S. (2009). Seeing the invisible: the scope and limits of unconscious processing in binocular rivalry. Prog. Neurobiol. 87, 195-211.

Logothetis, N. K. (1998). Single units and conscious vision. Philos. Trans. R. Soc. Lond. B Biol. Sci. 353, 1801-1818.

Lumer, E. D., Friston, K., and Rees, G. (1998). Neural correlates of perceptual rivalry in the human brain. Science 280, 1930-1934.

Meng, M., and Tong, F. (2004). Can attention selectively bias bistable perception? Differences between binocular rivalry and ambiguous figures. J. Vis. 4, 539-551.

Nguyen, V. A., Freeman, A. W., and Alais, D. (2003). Increasing depth of binocular rivalry suppression along two visual pathways. Vision Res. 43, 2003-2008.

Noest, A. J., van Ee, R., Nijs, M. M., and van Wezel, R. J. A. (2007) Percept-choice sequences driven by interrupted ambiguous stimuli: A low-level neural model. J. Vision 7(8):10, 1-14

Paffen, C. L. E., and Alais, D. (2011). Attentional modulation of binocular rivalry. Front. Hum. Neurosci. 5:105. doi: 10.3389/ fnhum.2011.00105

Paffen, C. L. E., Alais, D., and Verstraten, F. A. J. (2006). Attentions speeds binocular rivalry. Psychol. Sci. 17, 752-756.

Pearson, J., and Brascamp, J. (2008). Sensory memory for ambiguous vision. Trends Cogn. Sci. 12, 334-341.

Pearson, J., and Clifford, C. (2005). Mechanisms selectively engaged in rivalry: normal vision habituates, rivalrous vision primes. Vision Res. 45, 707-714.

Pearson, J., Tadin, D., and Blake, R. (2007). The effects of transcranial magnetic stimulation on visual rivalry. J. Vis. 7, 2.1-2.11.

Polonsky, A., Blake, R., Braun, J., and Heeger, D. J. (2000). Neuronal activity in human primary visual cortex correlates with perception during binocular rivalry. Nat. Neurosci. 3, 1153-1159.

Roeber, U., Veser, S., Schröger, E., and O'Shea, R. P. (2011). On the role of attention in binocular rivalry: electrophysiological evidence. PLoS One 6:e22612. doi: 10.1371/journal.pone.0022612
Seely, J., and Chow, C. (2011). Role of mutual inhibition in rivalry. J. Neurophysiol. 106, 2136-2150.

Shpiro, A., Moreno-Bote, R., Rubin, N., and Rinzel, J. (2009). Balance between noise and adaptation in competition models of perceptual bistabilty. J. Comput. Neurosci. 27, 37-54.

Stein, T., and Sterzer, P. (2011). Highlevel face adaptation depends on visual awareness: evidence from continuous flash suppression. J. Vis. 11, 5.1-14.

Sterzer, P., and Kleinschmidt, A. (2007). A neural basis for inference in perceptual ambiguity. Proc. Natl. Acad. Sci. U.S.A. 104, 323-328.

Sterzer, P., Kleinschmidt, A., and Rees, G. (2009). The neural bases of multistable perception. Trends Cogn. Sci. 13, 310-318.

Stuit, S. M., Paffen, C. L. E., van der Smagt, M. J., and Verstraten, F. A. J. (2011). What is grouping during binocular rivalry? Front. Hum. Neurosci. 5:117. doi: 10.3389/fnhum.2011.00117

Theodoni, P., Panagiotaropoulos, T. I., Kapoor, V., Logothetis, N., and Deco, G. (2011a). Cortical microcircuit dynamics mediating binocular rivalry: the role of adaptation and inhibition. Front. Hum. Neurosci. 5:145. doi: 10.3389/fnhum.2011.00145

Theodoni, P., Kovács, G., Greenlee, M. W., and Deco, G. (2011b). Neuronal adaptation effects in decision making. J. Neurosci. 31, 234-246.

van Boxtel, J. J. A., Alais, D., and van Ee, R. (2008). Retinotopic and non-retinotopic stimulus encoding in binocular rivalry and the involvement of feedback. J. Vis. 8 , 17.1-17.10.

van Ee, R. (2009). Stochastic variations in sensory awareness are driven by noisy neuronal adaptation: evidence from serial correlations in perceptual bistability. J. Opt. Soc. Am. A Opt. Image Sci. Vis. 26, 2612-2622.

van Ee, R. (2011). Percept-switch nucleation in binocular rivalry reveals local adaptation characteristics of early visual processing. J. Vis. $11,13.1-13.12$.

van Ee, R., van Boxtel, J. J. A. Parker, A. L., and Alais, D. (2009). Multisensory congruency as a mechanism for attentional control over perceptual selection. J. Neurosci. 29, 11641-11649.

van Ee, R., van Dam, L. C. J., and Brouwer, G. J. (2005). Voluntary control and the dynamics of perceptual bistability. Vision Res. 45, 41-55.

Wheatstone, C. (1838). Contributions to the physiology of vision - part the first. on some remarkable and hitherto unobserved phenomena of binocular vision. Philos. Trans. R. Soc. Lond. B Biol. Sci. 128, 371-394.

Wilson, H. R. (2003). Computational evidence for a rivalry hierarchy in vision. Proc. Natl. Acad. Sci. U.S.A. 100, 14499-14503.

Wilson, H. R. (2007). Minimal physiological conditions for binocular rivalry and rivalry memory. Vision Res. 47, 2741-2750.

Wolfe, J. (1986). Stereopsis and binocular rivalry. Psychol. Rev. 93, 269-282. Zaretskaya, N., Thielscher, A., Logothetis, N. K., and Bartels, A. (2010). Disrupting parietal function prolongs dominance durations in binocular rivalry. Curr. Biol. 20, 2106-2111.

Zhang, P., Jamison, K., Engel, S., He, B. and $\mathrm{He}, \mathrm{S}$. (2011). Binocular rivalry requires visual attention. Neuron 71 , 362-369.

Zhou, W., Jiang, Y., He, S., and Chen, D. (2010). Olfaction modulates visual perception in binocular rivalry. Curr. Biol. 20, 1356-1358.

Conflict of Interest Statement: The authors declare that the research was conducted in the absence of any commercial or financial relationships that could be construed as a potential conflict of interest.

Received: 01 August 2011; accepted: 14 February 2012; published online: 01 March 2012.

Citation: Roumani D and Moutoussis K (2012) Binocular rivalry alternations and their relation to visual adaptation. Front. Hum. Neurosci. 6:35. doi: 10.3389/fnhum.2012.00035

Copyright (c) 2012 Roumani and Moutoussis. This is an open-access article distributed under the terms of the Creative Commons Attribution Non Commercial License, which permits non-commercial use, distribution, and reproduction in other forums, provided the original authors and source are credited. 